RESEARCH ARTICLE

\title{
Amplification characteristics in active tapered segmented cladding fiber with large mode area
}

\author{
Caijian Xie ${ }^{1}$, Tigang Ning ${ }^{1}$, Jingjing Zheng ${ }^{1}, \mathrm{Li} \mathrm{Pei}^{1}$, Jianshuai Wang ${ }^{1}$, Jing $\mathrm{Li}^{1}$, Haidong You $^{2}$, \\ Chuangye Wang ${ }^{1}$, and Xuekai Gao ${ }^{1}$ \\ ${ }^{1}$ Key Laboratory of All Optical Network \& Advanced Telecommunication Network of EMC, Institute of Lightwave Technology, Beijing \\ Jiaotong University, Beijing 100044, China \\ ${ }^{2}$ Science and Information College, Qingdao Agricultural University, Qingdao 266109, China \\ (Received 5 January 2021; revised 16 March 2021; accepted 30 March 2021)
}

\begin{abstract}
A kind of tapered segmented cladding fiber (T-SCF) with large mode area (LMA) is proposed, and the mode and amplification characteristics of T-SCFs with concave, linear, and convex tapered structures are investigated based on finite-element method (FEM) and few-mode steady-state rate equation. Simulation results indicate that the concave tapered structure can introduce high loss for high-order modes (HOMs) that is advantageous to achieve single-mode operation, whereas the convex tapered structure provides large effective mode area that can help to mitigate nonlinear effects. Meanwhile, the small-to-large amplification scheme shows further advantages on stripping off HOMs, and the large-to-small amplification scheme decreases the heat load density induced by the high-power pump. Moreover, singlemode propagation performance, effective mode area, and heat load density of the T-SCF are superior to those of tapered step index fiber (T-SIF). These theoretical model and numerical results can provide instructive suggestions for designing high-power fiber lasers and amplifiers.
\end{abstract}

Keywords: bending loss; heat load density; high-order modes filtering; large mode area; tapered fibers

\section{Introduction}

Over recent decades, fiber lasers and amplifiers have attracted great interest owing to their advantages of high slope efficiency, high beam quality, superior thermal management property, and compactness ${ }^{[1]}$. Fiber lasers and amplifiers are widely used in the field of laser marking, material processing ${ }^{[2]}$, and many other industrial applications. Although the power of single-mode fiber laser system has already reached a high level of $10 \mathrm{~kW}^{[3]}$, scientific and industrial applications seek much higher laser power and light beam quality ${ }^{[4]}$. However, nonlinear effects induced by high laser power limit further scaling of the output power. For example, single-frequency or narrowlinewidth fiber lasers' power scaling is limited by stimulated Brillouin scattering (SBS), and power scaling for ultrashort

Correspondence to: J. Zheng, Key Laboratory of All Optical Network \& Advanced Telecommunication Network of EMC, Institute of Lightwave Technology, Beijing Jiaotong University, Beijing 100044, China. Email: jjzheng@bjtu.edu.cn pulsed fiber laser systems is limited by stimulated Raman scattering (SRS). Therefore, suppressing nonlinear effects is particularly critical for further scaling of the output power from high-power fiber lasers and amplifiers ${ }^{[5,6]}$. The most direct and effective approach to increase the threshold of nonlinear effects is to increase the effective mode area $\left(A_{\text {eff }}\right)$, and the simplest and most obvious way of increasing $A_{\text {eff }}$ is to increase the core diameter $(D)$ and decrease numerical aperture $(N A)$. Fibers that achieve large mode area (LMA) by increasing the core diameter and decreasing $N A$ are widely applied in high-power fiber laser system ${ }^{[7]}$. Such LMA fibers can effectively mitigate nonlinear effects ${ }^{[8]}$, but there are two emerging problems in practical applications. One is that they support high-order modes (HOMs) owing to the larger core diameter and lower $N A$, which results in transverse mode instability (TMI) at high average power levels ${ }^{[9]}$; the other is that such fibers become highly sensitive to bending, which results in high bending loss and severe mode area compression. Therefore, it is important for LMA fibers to suppress nonlinear effects and ensure transverse mode stability simultaneously. 
Over the past decade, varieties of LMA fibers have been proposed to scale output power and ensure high beam quality in high-power fiber laser and amplifier systems. The novel LMA fibers include photonic crystal fiber $(\mathrm{PCF})^{[10]}$, leakage channel fiber $(\mathrm{LCF})^{[11]}$, rod-type fiber ${ }^{[12]}$, low-NA step index fiber $(\mathrm{SIF})^{[13]}$, segmented cladding fiber $(\mathrm{SCF})^{[14,15]}$, and other promising LMA fibers ${ }^{[16,17]}$. Much effort has been directed towards developing novel fiber designs that offer LMA and single-mode operation. For example, SIF obtains LMA by increasing core radius and decreasing $N A$, and single-mode propagation by bend-induced HOMs filtering. PCF and LCF with special fiber structures show good potential for mode area scaling and single-mode operation, but their performances become worse under bending. SCF, first reported by Rastogi and Chiang in $2001^{[14]}$, is characterized by its cladding that has periodic distribution of high and low refractive indices in the angular direction, and it can have both large core and high NA. In 2004, the large-core SCFs made from polymer material were first demonstrated by Yeung et al. ${ }^{[18]}$, and the large-core four-segment and eightsegment SCFs were fabricated by the cladding-segmentin-tube technique and cladding-core-segment-in-tube technique, respectively. In 2015, Hooda et al. ${ }^{[19]}$ demonstrated fabrication of a silica-based SCF by using the stack-and-draw technique, and the fabricated SCF showed efficient HOMs filtering. HOMs propagating in SCF can be stripped off by taking advantage of high leakage loss difference between fundamental mode and them. Therefore, large-core SCF can simultaneously support LMA and single-mode operation, which is attractive for high-power fiber lasers and amplifiers.

In addition to these longitudinal uniform LMA fibers, a novel promising tapered fiber design has been developed in recent years. Different from the longitudinal uniform fibers, core and cladding diameters of tapered fibers vary along the fiber length. In high-power fiber laser and amplifier systems, tapered LMA fibers exhibit numerous unique advantages compared with regular LMA fibers, such as higher absorption per unit length, intrinsic built-in mechanism for amplified spontaneous emission (ASE) suppression, and a high factor of brightness. In 2015, a polarization-maintaining Yb-doped tapered fiber was applied to an ultrashort pulse fiber laser system ${ }^{[20]}$. The tapered fiber with a single-mode input end (core and cladding diameters of 10 and $80 \mu \mathrm{m}$ ) and a multimode output end (core and cladding diameters of 45 and $430 \mu \mathrm{m}$ ) was used as the output amplifier stage to reach the megawatt output power. In 2016, a tapered double-clad fiber (T-DCF) was demonstrated and used as gain fiber in the continuous wave $(\mathrm{CW})$ high-power amplifier system, actively $Q$-switched fiber laser, and picosecond pulsed fiber laser system. ${ }^{[21]}$ In 2017, LMA Yb-doped tapered fiber fabricated by a non-stationary fiber drawing process was employed in a high-power fiber amplifier system, which ensured a low ASE level and realized a high peak power of $22 \mathrm{MW}^{[22]}$. In 2018, a birefringent Yb-doped tapered fiber was demonstrated for linearly polarized $\mathrm{CW}$ and pulsed high-power amplifiers. ${ }^{[23]}$ In 2019, the double cladding tapered $\mathrm{Yb}$-doped fiber was used in a high-power monolithic fiber laser oscillator, which obtained the maximum average power of $1720 \mathrm{~W}$ when employing $975 \mathrm{~nm}$ pump source, ${ }^{[24]}$ but the TMI effect occurred at the output power of $1350 \mathrm{~W}$ when employing $915 \mathrm{~nm}$ pump.

In this paper, a tapered segmented cladding fiber (T-SCF) is proposed. Modal loss, effective mode area, power evolution, and heat load density of T-SCFs with concave, linear, and convex tapered structures are theoretically investigated based on a finite-element method (FEM) and few-mode steady-state rate equation. The simulation consists of three parts. In the first part, mode characteristics of straight and bent T-SCFs with different parabolic shape factors $(b)$ are investigated by the FEM. In the second part, we set the small end of the T-SCF as the pump and signal input end, and simulate the amplification characteristics of T-SCFs based on a simple amplifier model and a few-mode steady-state rate equation. In addition, we compare laser performance of the amplifier based on T-SCF with that of the amplifier based on tapered step index fiber (T-SIF). In the third part, we choose the large end of the T-SCF as the pump and signal input end, and study the laser performances of the amplifier based on T-SCF. Results show that the concave tapered structure can provide high modal loss for HOMs, whereas the convex tapered structure presents large effective mode area. Thus, concave T-SCF has advantages on stripping off HOMs but convex T-SCF can mitigate nonlinear effects more effectively. Single-mode operation is easier to achieve for TSCF under small-to-large amplification scheme, whereas heat load density of T-SCF under large-to-small amplification scheme is much lower than that under small-to-large amplification scheme. These results suggest that the tapered structure and amplification scheme have an effect on the mode characteristics and laser performance, which provides theoretical foundations for developing compact high-power fiber lasers and amplifiers.

\section{Theory and model}

The schematic of the SCF is displayed in Figure 1. Parameters $a$ and $b$ are the core and cladding radii, respectively, and $\varphi$ is the azimuth angle between the $x$-axis and bending orientation. The fiber comprises a uniform core region $(0<r<a)$ with high refractive index $\left(n_{1}\right)$ and a segmented cladding region $(a<r<b)$. The low-index $\left(n_{2}\right)$ segments and highindex $\left(n_{1}\right)$ segments alternate periodically and angularly, and their angular widths are $\theta_{1}$ and $\theta_{2}$. The period of the segment cladding is defined as $\Lambda=\theta_{1}+\theta_{2}$, and $N$ is the number of the fiber cladding segments. The refractive index difference between $n_{1}$ and $n_{2}$ is characterized by $\Delta=\left(n_{1}^{2}-n_{2}^{2}\right) / 2 n_{1}^{2}$ and we assume $\Delta \ll 1$, so the SCF can be considered as weakly guiding fiber. The transverse component of the elec- 

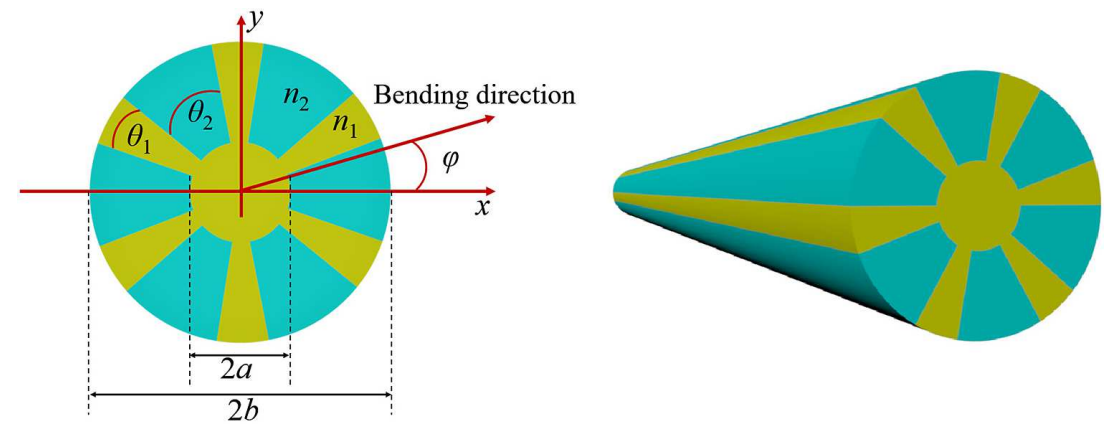

Figure 1. Schematic diagram of the SCF, $N=6^{[25]}$.

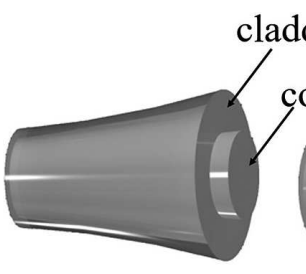

(a) $b_{\mathrm{f}}<b_{0}$

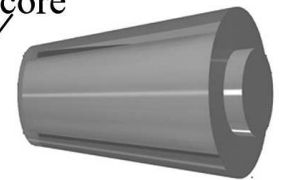

(b) $b_{\mathrm{f}}=b_{0}$

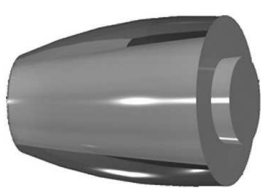

(c) $b_{\mathrm{f}}>b_{0}$

Figure 2. Three major tapered categories: (a) concave tapered fiber, (b) linear tapered fiber, and (c) convex tapered fiber.

tromagnetic field of the weakly guiding fiber satisfies the scalar wave equation. The scalar wave equation for SCF can be solved by the radial effective-index method (REIM) and $\mathrm{FEM}^{[26]}$ and the transverse mode characteristics are simulated by FEM in this paper.

Tapered fibers include the fast-varying tapered fiber and slowly varying tapered fiber. The length of fast varying tapered fibers is generally short (even in the millimeter range $)^{[26]}$, while the length of slowly varying tapered fiber ranges from several to hundreds of meters, and Figure 2 displays three novel tapered structures ${ }^{[27,28]}$. In this paper, our numerical simulation concerns on the long T-SCF that has a longitudinally varying core/cladding radius along the fiber length, and the core radius profile of the T-SCF can be given by ${ }^{[29]}$

$$
\rho(z)=\frac{b_{0}-b_{\mathrm{f}}}{2 L} z^{2}+\frac{b_{\mathrm{f}}}{2} z+\frac{1}{2} D_{1},
$$

where $D_{1}$ and $D_{2}$ are the core diameters of the small and large end, respectively, $L$ is the length of T-SCF, $b_{\mathrm{f}}$ is the parabolic shape factor, and $b_{0}=\left(D_{2}-D_{1}\right) / L$ is average taper angle. According to the difference between $b_{\mathrm{f}}$ and $b_{0}$, the radius profile can be divided into three categories shown in Figure 3, which include $b_{\mathrm{f}}<b_{0}, b_{\mathrm{f}}=b_{0}$, and $b_{\mathrm{f}}>b_{0}$ corresponding to concave, linear, and convex radius profile.

Longitudinal stretching along the fiber length results in elasto-optical effect when the fiber is curved, which makes the refractive index change. We take into account refractive index perturbations induced by bending, and employ

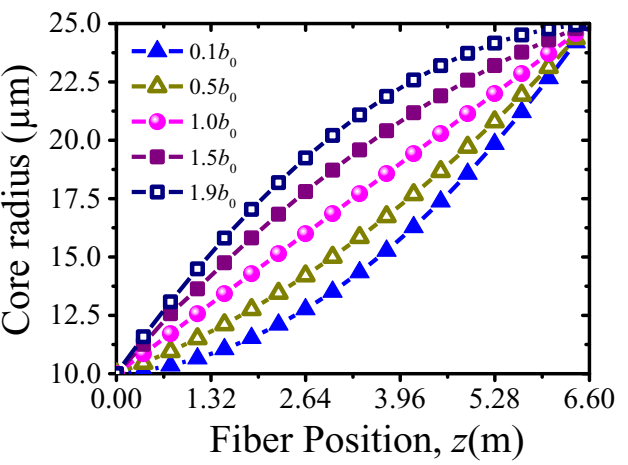

Figure 3. Effects of different parabolic shape factors on the core radius profile (from the small end to the large end).

coordinate transformation that allows the bent fiber to be represented by an equivalent, straight fiber with modified refractive index distribution. Considering the non-circular symmetry and tapered structure of SCF, the bend-induced refractive index should be $\varphi$-dependent and $z$-dependent, and it takes the form ${ }^{[15]}$

$$
n_{\text {bent }}(x, y, z)=n_{\text {straight }}(x, y, z)\left(1+\frac{\vec{x} \cos \varphi+\vec{y} \sin \varphi}{\rho R}\right) \text {, }
$$

where $n_{\text {straight }}(x, y, z)$ is the index profile of the straight fiber, $R$ is the bending radius, and $\vec{x}$ and $\vec{y}$ denote the orientation of Cartesian coordinates. Note that $\rho$ is fixed to 1.25 when taking account of the stress factor for the silicabased fiber. ${ }^{[15]}$

In optical fibers, the modal field actually distributes both in the core and in cladding. The leakage structure of SCF suggests that modal fields may have more distribution in cladding, and thus modal fields would experience greater leakage loss. In addition, bend-induced stress can have a significant effect on leakage loss. In high-power laser and amplifier systems, it can achieve single-mode operation by taking advantages of leakage loss characteristics. In this paper, we focus on the modal leakage loss aiming at achieving HOMs filtering by coiling fiber. Based on Equations (1) and (2), we can determine the LP modes of SCF and simulate 
their bend-induced characteristics by FEM. The effective index of the $i$ th mode $\left(n_{\text {eff }}^{\mathrm{s}, i}\right)$ becomes complex with the real part and the imaginary part under FEM, and the imaginary part is related to the modal loss that can be expressed as ${ }^{[30]}$

$$
\alpha_{\mathrm{s}, i}(Z)=\frac{20}{\operatorname{In} 10} \frac{2 \pi}{\lambda} \operatorname{Im}\left(n_{\mathrm{eff}}^{\mathrm{s}, i}\right)
$$

As in the previous work ${ }^{[31]}$, we assume an additional layer known as a perfectly matched layer (PML) in the outer boundary region to absorb the leakage light from the cladding when simulating mode loss. In reality, high absorption for leakage light in the cladding can be ensured by employing the cladding light stripper.

We propose a simple amplifier model based on the TSCF, and then there are two amplification schemes owing to the different signal input end. A large-to-small amplification scheme denotes that the large end of the T-SCF is the signal input end, whereas a small-to-large amplification scheme means that the small end of the T-SCF is the signal input end. Based on the $z$-dependent transverse mode parameters calculated by FEM, then amplification characteristics can be simulated by the few-mode steady-state rate equation:

$$
\begin{aligned}
& \frac{N_{2}(z)}{N_{\mathrm{Yb}}}=\left\{\frac{\left[P_{\mathrm{p}}^{+}(z)+P_{\mathrm{p}}^{-}(z)\right] \sigma_{\mathrm{ap}} \Gamma_{\mathrm{p}}(z)}{h v_{\mathrm{p}} A_{\mathrm{dope}}(z)}\right. \\
&\left.+\sum \frac{\left[P_{\mathrm{s}, i}^{+}(z)+P_{\mathrm{s}, i}^{-}(z)\right] \sigma_{\mathrm{as}} \Gamma_{\mathrm{s}, i}(z)}{h v_{\mathrm{s}} A_{\mathrm{dope}}(z)}\right\} \\
& \times\left\{\frac{\left[P_{\mathrm{p}}^{+}(z)+P_{\mathrm{p}}^{-}(z)\right]\left(\sigma_{\mathrm{ap}}+\sigma_{\mathrm{ep}}\right) \Gamma_{\mathrm{p}}(z)}{h v_{\mathrm{p}} A_{\mathrm{dope}}(z)}\right. \\
&\left.+\sum \frac{\left[P_{\mathrm{s}, i}^{+}(z)+P_{\mathrm{s}, i}^{-}(z)\right]\left(\sigma_{\mathrm{as}}+\sigma_{\mathrm{es}}\right) \Gamma_{\mathrm{s}, i}(z)}{h v_{\mathrm{s}} A_{\mathrm{dope}}(z)}+\frac{1}{\tau}\right\}^{-1} \\
& \pm \frac{\mathrm{d} P_{\mathrm{p}}^{ \pm}(z)}{\mathrm{d} z}=\Gamma_{\mathrm{p}}(z)\left[\sigma_{\mathrm{ep}} N_{2}(z)-\sigma_{\mathrm{ap}} N_{1}(z)\right] P_{\mathrm{p}}^{ \pm}(z) \\
&-\frac{\ln 10}{10} \alpha_{\mathrm{p}}(z) P_{\mathrm{p}}^{ \pm}(z), \\
& \pm \frac{\mathrm{d} P_{\mathrm{s}, i}^{ \pm}(z)}{\mathrm{d} z}=\left[\sigma_{\mathrm{es}} N_{2}(z)-\sigma_{\mathrm{as}} N_{1}(z)\right] \Gamma_{\mathrm{s}, i}(z) P_{\mathrm{s}, i}^{ \pm}(z) \\
&-\frac{\ln 10}{10} \alpha_{\mathrm{s}, i}(z) P_{\mathrm{s}, i}^{ \pm}(z),
\end{aligned}
$$

where $N_{1} / N_{2}$ and $N_{\mathrm{Yb}}$ are the lower/upper state population and $\mathrm{Yb}^{3+}$ ion concentration, respectively; $P_{\mathrm{p} / \mathrm{s}, i}^{+}$and $P_{\mathrm{p} / \mathrm{s}, i}^{-}$ represent the forward and backward pump/ith modal power; $\sigma_{\text {ap }} / \sigma_{\text {ep }}$ and $\sigma_{\text {as }} / \sigma_{\text {es }}$ are the absorption/emission crosssections at the pump and signal wavelengths; $\alpha_{\mathrm{p}}(z)$ and $\alpha_{\mathrm{s}, i}(z)$ are the pump loss and $i$ th mode loss that can be calculated according to Equation (3), and the units for those losses are $\mathrm{dB} / \mathrm{m}$; the pump and $i$ th mode power fill factor
Table 1. The initial simulation parameters.

\begin{tabular}{cccc}
\hline Parameter & Value & Parameter & Value \\
\hline$n_{1}$ & 1.449 & $\Lambda$ & $\pi / 3$ \\
$n_{2}$ & 1.444 & $L$ & $6.6 \mathrm{~m}$ \\
$D_{1}$ & $20 \mu \mathrm{m}$ & $N_{\mathrm{Yb}}$ & $2 \times 10^{25} \mathrm{~m}^{-3}$ \\
$D_{2}$ & $50 \mu \mathrm{m}$ & $\tau$ & $800 \mathrm{~ms}$ \\
$b_{1}$ & $62.5 \mu \mathrm{m}$ & $\lambda_{\mathrm{S}}$ & $1060 \mathrm{~nm}$ \\
$\theta_{1}$ & $\pi / 9$ & $\lambda_{\mathrm{P}}$ & $975 \mathrm{~nm}$ \\
\hline
\end{tabular}

are denoted by $\Gamma_{\mathrm{p}}(z)$ and $\Gamma_{\mathrm{s}, i}(z)$; and $A_{\mathrm{dope}}(z)$ is the effective ion-doped area that is mainly dependent on Equation (1).

The rare-earth-doped core can act as a heat source under a high-power pump ${ }^{[32]}$, and thus the longitudinally varying core radius of T-SCF results in longitudinally varying heat load density that is

$$
q=\frac{Q(z)}{A_{\text {dope }}(z)}=\frac{\left(\frac{\mathrm{d} P_{\mathrm{p}}^{-}}{\mathrm{d} z}-\frac{\mathrm{d} P_{\mathrm{p}}^{+}}{\mathrm{d} z}\right) \times\left(1-\frac{\lambda_{\mathrm{p}}}{\lambda_{\mathrm{s}}}\right)}{A_{\text {dope }}(z)},
$$

where $q$ and $Q$ are the heat load density and heat load.

Both fiber radius profile and refractive index distribution are $z$-dependent that can induce the mode characteristics to evolve along the T-SCF, and thus laser performance can present evolution behaviors. In the following section, we analyze the mode and laser evolution characteristics of SCF with different tapered structures.

\section{Results and discussion}

This section consists of three parts. In the first part, we simulate fiber core radius and modal loss characteristics that change along T-SCF according to Equations (1)-(3). In the second part, the laser characteristics of the T-SCF under a large-to-small amplification scheme are analyzed by substituting the mode parameters calculated in the first part into Equations (4)-(7), and are compared with that of the T-SIF under a large-to-small amplification scheme. In the third part, laser characteristics of the T-SCF under a smallto-large amplification scheme are discussed. Assuming the T-SCF is doped with ytterbium ions, we choose the cladding radius in the small end of the fiber $b_{1}=62.5 \mu \mathrm{m}$ and keep the cladding-core radius ratio as 6.25 . Then we preliminarily optimize the fiber parameters to ensure that the loss of $\mathrm{LP}_{01}$ of T-SCF is lower than $0.1 \mathrm{~dB} / \mathrm{m}^{[33]}$ and to ensure saturation amplification for $\mathrm{LP}_{01}$ mode. The initial simulation parameters are listed in Table 1.

\subsection{Mode characteristics of T-SCF}

Figure 3 clearly demonstrates the core radius profile for different tapered structures. Note that the legends in the figure mean the proportional relationship between the parabolic 

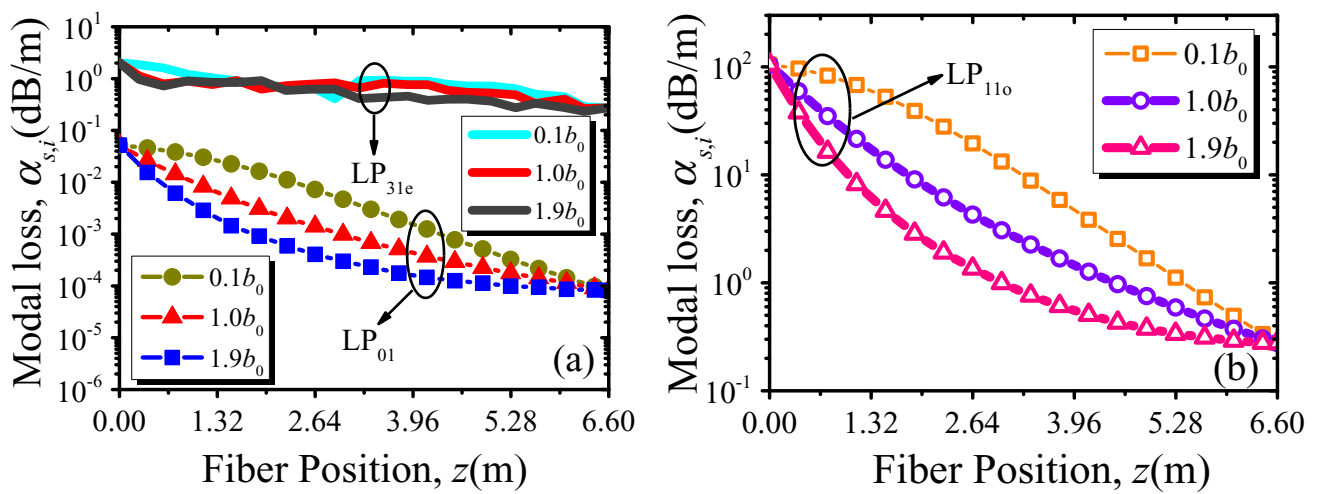

Figure 4. Comparison of modal loss of straight T-SCF from the small end to the large end: (a) mode losses of $\mathrm{LP}_{01}$ and $\mathrm{LP}_{31 \mathrm{e}}$; (b) mode loss of $\mathrm{LP}_{110}$.
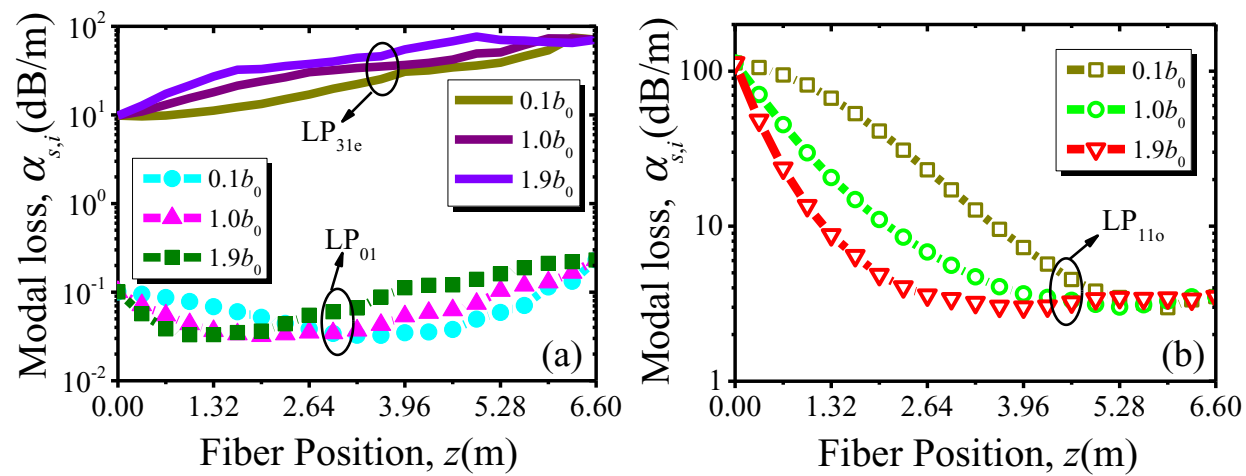

Figure 5. Comparison of modal loss of T-SCF with a bending radius of $32 \mathrm{~cm}$ : (a) mode losses of $\mathrm{LP}_{01}$ and $\mathrm{LP}_{31 \mathrm{e}}$; (b) mode loss of $\mathrm{LP}_{110}$.

shape factor $b_{\mathrm{f}}$ and the average taper angle $b_{0}$. For example, $0.1 b_{0}$ represents the relational expression $b_{\mathrm{f}}=0.1 b_{0}$. It is obvious that T-SCF with a larger parabolic shape factor has a larger core radius at the same fiber position. Therefore, TSCF with a larger parabolic shape factor would be better for enlarging mode area. These characteristics of the fiber structure may have an effect on the T-SCF-based laser and amplifier performance. On the one hand, LMA is helpful for mitigating nonlinear effects. On the other hand, the large core radius may reduce HOMs loss that has a negative effect on single-mode operation. In the following, we analyze the mode loss, amplification characteristics, and single-mode propagation for T-SCF with different parabolic shape factor. We choose $0.1 b_{0}, 1 b_{0}$, and $1.9 b_{0}$ to denote the concave, linear, and convex T-SCF, respectively.

Based on initial parameters and keeping the clad-to-core ratio as a constant of 6.25 , we simulate the mode loss of straight and bent T-SCF using FEM. Simulation results show that mode losses of $\mathrm{LP}_{11}\left(\mathrm{LP}_{11 \mathrm{o}}\right.$ and $\left.\mathrm{LP}_{11 \mathrm{e}}\right)$ and $\mathrm{LP}_{31 \mathrm{e}}$ are lower than the losses of other HOMs, where the subscripts 'e' or ' $\mathrm{o}$ ' added to the LP modal labels denote that the mode is even or odd with respect to the $y$-axis. For example, the minimum losses of $\mathrm{LP}_{11 \mathrm{o}}$ and $\mathrm{LP}_{31 \mathrm{e}}$ are 0.346 and $0.247 \mathrm{~dB} / \mathrm{m}$, respectively, whereas the minimum loss of $\mathrm{LP}_{21}$ is as high as $38.6 \mathrm{~dB} / \mathrm{m}$ along the T-SCF. Such high mode losses can make those HOMs be stripped off easily. Therefore, we focus on the mode and amplification characteristics of $\mathrm{LP}_{01}, \mathrm{LP}_{11}$, and $\mathrm{LP}_{31 \mathrm{e}}$ in the following analyses. Figure 4 displays the mode loss evolution for $\mathrm{LP}_{01}$, $\mathrm{LP}_{11 \mathrm{o}}$, and $\mathrm{LP}_{31 \mathrm{e}}$. Significantly, the reason why only mode loss of $\mathrm{LP}_{11}$ is shown in the figure of the $\mathrm{LP}_{11}$ mode is that mode losses of $\mathrm{LP}_{11}$ and $\mathrm{LP}_{11}$ are almost the same. According to Figure 4, mode losses of $\mathrm{LP}_{01}, \mathrm{LP}_{11}$, and $\mathrm{LP}_{31 \mathrm{e}}$ gradually drop from the small end to the large end of T-SCF, and a larger parabolic shape factor results in lower modal loss. The mode loss of $\mathrm{LP}_{11 \text { o }}$ decreases from $104.88 \mathrm{~dB} / \mathrm{m}$ at the small end of T-SCF to $0.28 \mathrm{~dB} / \mathrm{m}$ at the large end, showing great decline. The mode loss of $\mathrm{LP}_{31 \mathrm{e}}$ drops from 2.03 to $0.27 \mathrm{~dB} / \mathrm{m}$, representing a slight decrease. More importantly, the loss of $\mathrm{LP}_{31 \mathrm{e}}$ is lower than that of $\mathrm{LP}_{11}$ owing to the symmetry between six modal lobes and six lowindex cladding segments. These results suggest that losses of lower-order modes are more sensitive to enlarging core radius than those of the higher-order modes.

Coiling fiber is a successful strategy to achieve effective single-mode operation for few-mode fibers because it introduces high losses for HOMs. Assuming the T-SCF is curved in the $x$-axis, we investigate mode loss under a bending radius of $32 \mathrm{~cm}$. Figure 5 illustrates the modal loss of the bent T-SCF; it is obvious that bending operation induces mode losses to increase significantly, especially loss of $\mathrm{LP}_{31 \mathrm{e}}$. Furthermore, bend-induced modal loss evolutions of $\mathrm{LP}_{01}$, 

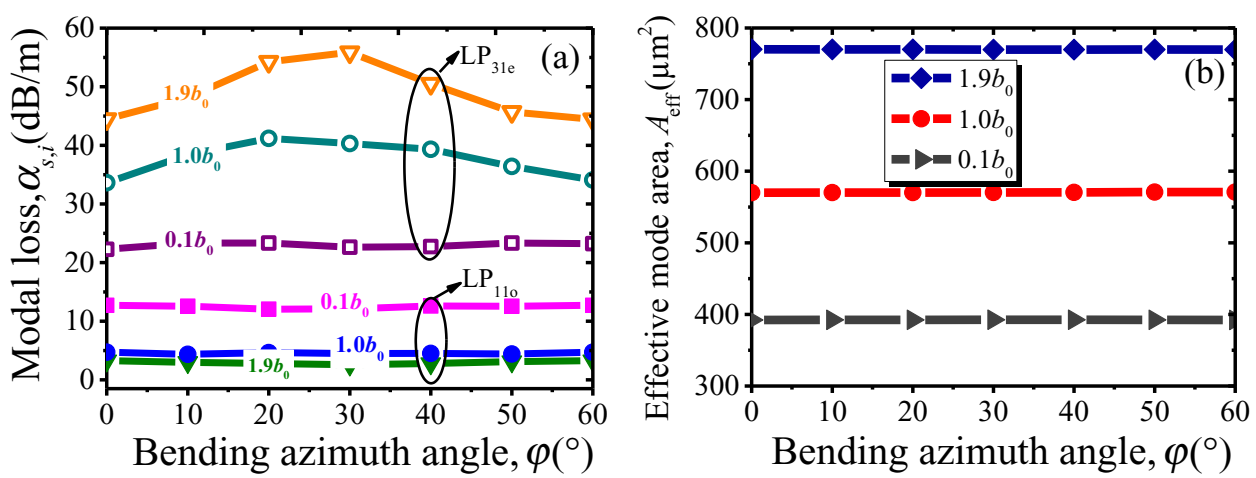

Figure 6. (a) Modal loss and (b) effective mode area of $\mathrm{LP}_{01}$ for T-SCF under various bending azimuth angles, $R=32 \mathrm{~cm}$, and $z=3.3 \mathrm{~m}$.

$\mathrm{LP}_{110}$, and $\mathrm{LP}_{31 \mathrm{e}}$ are different from each other. From the small end to the large end, loss of $\mathrm{LP}_{01}$ decreases to the minimum value and then increases gradually. Loss of $\mathrm{LP}_{31 \mathrm{e}}$ increases from 9.81 to $70.77 \mathrm{~dB} / \mathrm{m}$, which indicates that the total loss of $\mathrm{LP}_{31 \mathrm{e}}$ is higher than $64.75 \mathrm{~dB}$ for the $6.6 \mathrm{~m}$ long T-SCF. In contrast to loss of $\mathrm{LP}_{31 \mathrm{e}}$, loss of $\mathrm{LP}_{11 \mathrm{o}}$ decreases from 113.01 to $3.51 \mathrm{~dB} / \mathrm{m}$. Based on these modal losses of the straight and bent T-SCF, we can simulate the amplification characteristics for the based T-SCF amplifier and analyze the laser performance.

Equations (2) and (3) suggest that bending azimuth angle may have an effect on mode characteristics. The elastooptical effect of the bent fiber is mainly induced by longitudinal stretching, and thus we can analyze the bending modal loss and mode area for a given $z$. Mode performance under bending azimuth angle in the range of $\left[0^{\circ}, 60^{\circ}\right]$ can fully represent overall performance of the fiber because the segment cladding period of the proposed SCF is $\pi / 3$. Figure 6 displays the effects of bending orientation on the mode performance at $R=32 \mathrm{~cm}$ and $z=3.3 \mathrm{~m}$. Loss of $\mathrm{LP}_{01}$ remains lower than $0.1 \mathrm{~dB} / \mathrm{m}$, not shown in the figure. Figure 6 clearly illustrates that the fiber performance is insensitive to the bending azimuth angle, which makes it more flexible and convenient when coiling fiber to achieve single-mode operation in actual applications.

\subsection{Amplification characteristics based on the small-to- large scheme}

In this section, a simple amplifier model is proposed to investigate laser characteristics under a small-to-large amplification scheme, as shown in Figure 7. We assume that the input pump power and signal power of $\mathrm{LP}_{01}$ are $1000 \mathrm{~W}$ and $10 \mathrm{~W}$, respectively, while those of $\mathrm{LP}_{11}\left(\mathrm{LP}_{11 \mathrm{o}}\right.$ and $\left.\mathrm{LP}_{11 \mathrm{e}}\right)$ and $\mathrm{LP}_{31 \mathrm{e}}$ are all $1 \mathrm{~W}$. Results show that the output powers of $\mathrm{LP}_{01}$ in the large end of the concave, linear, and convex T-SCF are $881.48,875.66$, and $872.42 \mathrm{~W}$, respectively. Thus, the larger parabolic shape factor may degrade the laser efficiency of $\mathrm{LP}_{01}$ mode. Figure 8(a) demonstrates output power of $\mathrm{LP}_{11}$. For concave and linear T-SCF, the output power of

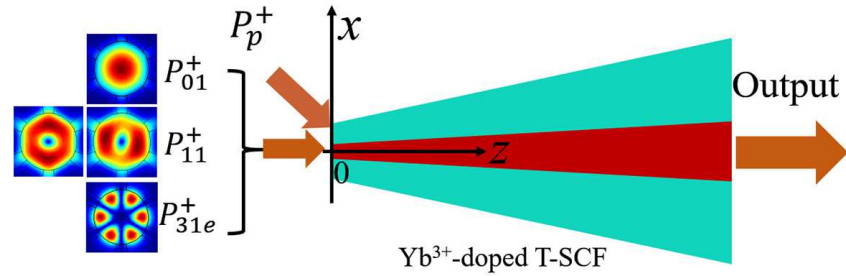

Figure 7. The amplifier model based on T-SCF under the small-to-large amplification scheme (the doped region colored red).

$\mathrm{LP}_{11}$ is lower than $1 \times 10^{-4} \mathrm{~W}$, which is extremely low when compared with the output power of $\mathrm{LP}_{01}$. Therefore, it can be considered that the $\mathrm{LP}_{11}$ mode in concave and linear T-SCF is stripped off successfully. The power of $\mathrm{LP}_{11}$ mode in convex T-SCF remains at around $0.1 \mathrm{~W}$ so can be considered as a noise signal. In addition to $\mathrm{LP}_{01}$ mode, $\mathrm{LP}_{31 \mathrm{e}}$ mode gets effective amplification owing to its low loss, as displayed in Figure 8(b). Moreover, the output power of $\mathrm{LP}_{31 \mathrm{e}}$ increases with the increasing parabolic shape factor, and is $24.69,31.29$, and $37.02 \mathrm{~W}$ for concave, linear, and convex T-SCF, respectively. These simulated results indicate that the concave T-SCF presents excellent HOMs filtering effect, but its effective mode area and thermal performance are inferior to that of linear and convex T-SCF, as illustrated in Figure 9. For a given fiber position $z$, for example, $z=4.0 \mathrm{~m}, A_{\mathrm{eff}}$ of $\mathrm{LP}_{01}$ and the heat load density in concave T-SCF are $486.49 \mu \mathrm{m}^{2}$ and $7.98 \times 10^{10} \mathrm{~W} / \mathrm{m}^{3}$, whereas those in the convex T-SCF are $904.45 \mu \mathrm{m}^{2}$ and $1.57 \times 10^{9} \mathrm{~W} / \mathrm{m}^{3}$. These comparisons indicate that convex T-SCF can help to mitigate nonlinear effects and thermal effects in high-power fiber lasers and amplifiers owing to its LMA and low heat load density.

We investigate modal power evolution of the linear T-SIF to make comparisons with that of T-SCF. Mode losses of the guided modes in straight linear T-SIF are lower than $1 \times 10^{-10} \mathrm{~dB} / \mathrm{m}$. Thus, those guided modes are effectively amplified owing to the low losses. The output power of $\mathrm{LP}_{01}$ in linear T-SIF is $725.0 \mathrm{~W}$, which is much lower than the $875.66 \mathrm{~W}$ of linear T-SCF. Although the output power of HOMs in linear T-SIF is higher than that in linear T-SCF, 

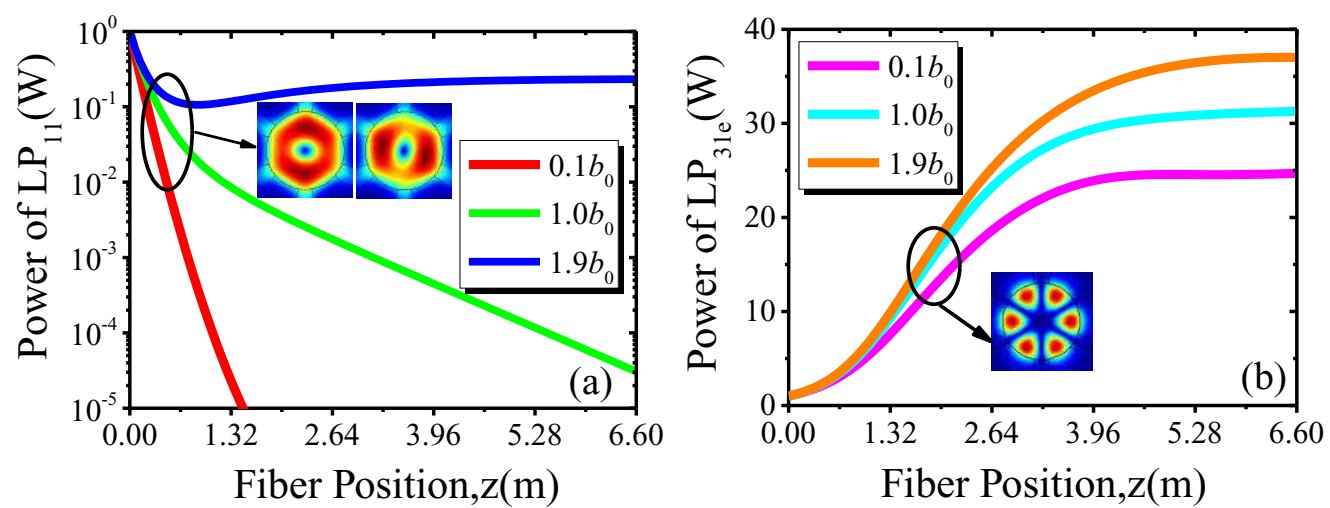

Figure 8. Modal power evolution of (a) $\mathrm{LP}_{11}$ mode and (b) $\mathrm{LP}_{31 \mathrm{e}}$ mode for concave, linear, and convex T-SCF based on the small-to-large amplification scheme.
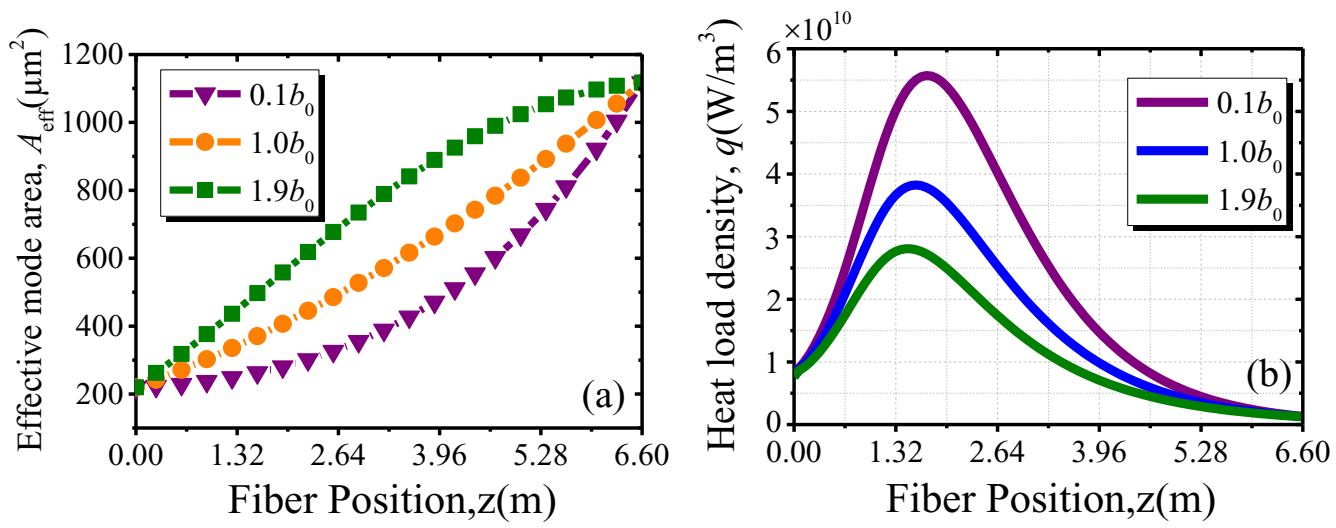

Figure 9. (a) Effective mode area of $\mathrm{LP}_{01}$ and (b) heat load density evolution along T-SCF.
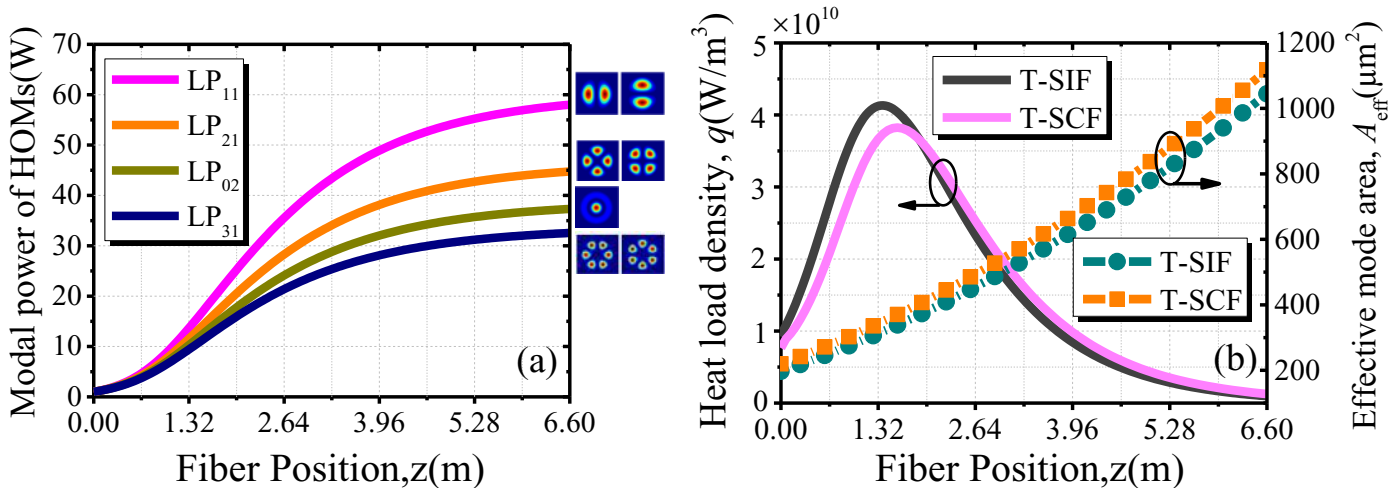

Figure 10. (a) Modal power evolution of four HOMs in linear T-SIF and (b) comparison of heat load density and effective mode area of $\mathrm{LP}_{01}$ between linear T-SCF and T-SIF.
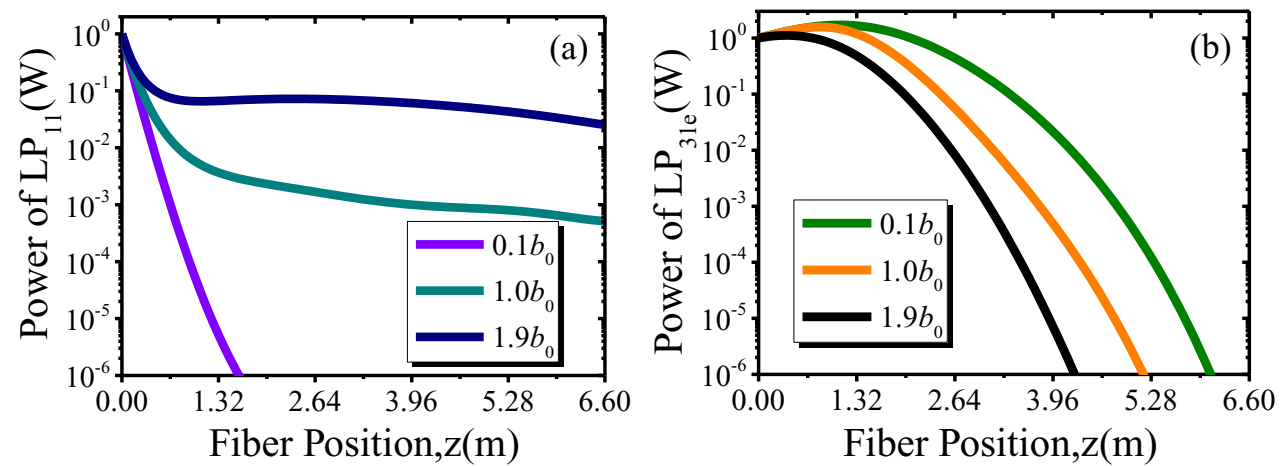

Figure 11. Power evolution of (a) $\mathrm{LP}_{11}$ mode and (b) $\mathrm{LP}_{31 \mathrm{e}}$ mode in the $\mathrm{T}-\mathrm{SCF}$ under a bending radius of $32 \mathrm{~cm}$. 
and Figure 10(a) displays the power evolution of four HOMs, it is clear that those HOMs are amplified effectively, and thus the T-SIF supports few-modes amplification. Figure 10(b) compares the heat load density and mode area in straight TSIF and T-SCF. The effective mode area of $\mathrm{LP}_{01}$ in T-SIF is smaller by $23.05-72.5 \mu \mathrm{m}^{2}$ than that of T-SCF, whereas the heat load density in the front-end of straight T-SIF is slightly higher than that in T-SCF.

To demonstrate the single-mode operation by coiling fiber, we simulate the bending-induced amplification characteristics for the T-SCF under a bending radius of $32 \mathrm{~cm}$ by keeping the input pump and signal power unchanged. The output powers of $\mathrm{LP}_{01}$ from the concave, linear, and convex T-SCF are $882.84,878.18$, and $861.02 \mathrm{~W}$. The output power

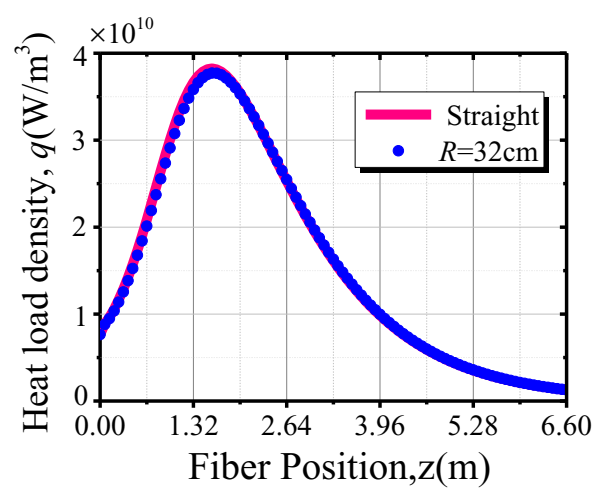

Figure 12. Comparison of heat load density between straight T-SCF and bent T-SCF of $R=32 \mathrm{~cm}$

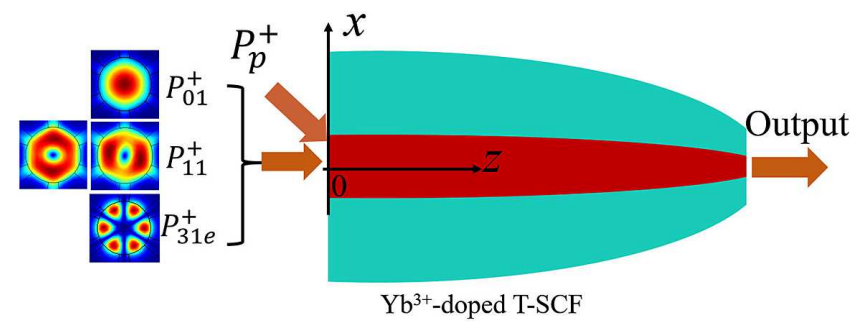

Figure 13. The amplifier model based on T-SCF under the large-to-small amplification scheme (the doped region colored red).

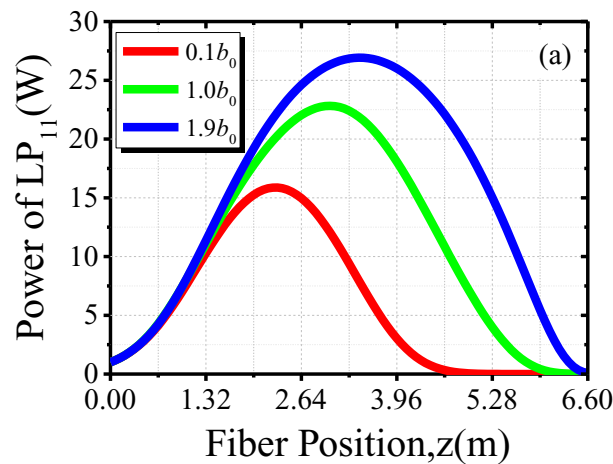

of $\mathrm{LP}_{11}$ from the concave T-SCF is lower than $1.0 \times 10^{-6} \mathrm{~W}$, which suggests that $\mathrm{LP}_{11}$ mode is filtered out effectively, as shown in Figure 11(a). As the output power of $\mathrm{LP}_{11}$ from linear and convex T-SCF still remains at the milliwatt level, the $\mathrm{LP}_{11}$ mode can thus work as noise. Figure 11(b) demonstrates that the signal power of $\mathrm{LP}_{31 \mathrm{e}}$ drops from $1 \mathrm{~W}$ to lower than the level of $10^{-6} \mathrm{~W}$, indicating that $\mathrm{LP}_{31 \mathrm{e}}$ mode is effectively stripped off. These results indicate that singlemode operation can be achieved by coiling T-SCF.

Comparison of heat load density between straight T-SCF and bent T-SCF is presented in Figure 12. It is clear that the bending operation $(R=32 \mathrm{~cm})$ has a minor effect on the heat load density. As illustrated in Equation (7), heat load density is directly dependent on the effective ion-doped area and state rate equation, but the effective ion-doped area and the state rate equation are hardly influenced by the loose bending radius. This is why heat load density has no significant difference between the straight and bent T-SCF.

\subsection{Amplification characteristics based on the large-to- small scheme}

The signal of HOMs may be effectively amplified owing to their low mode losses. We set the large end of the fiber as the input end to investigate the laser characteristics of the straight and bent T-SCF, as illustrated in Figure 13. The output powers of $\mathrm{LP}_{01}$ from the concave, linear, and convex T-SCF are 848.53, 842.66, and 841.76 W, respectively, which suggests the concave structure can help to maintain high amplification efficiency for $\mathrm{LP}_{01}$ mode. Signal of $\mathrm{LP}_{11}$ mode under the large-to-small scheme is effectively amplified in the middle section of the fiber, as displayed in Figure 14(a). The low loss in the front segment of T-SCF under the large-to-small scheme results in high gain for the signal of $\mathrm{LP}_{11}$, but $\mathrm{LP}_{11}$ gradually leaks out when propagating to the rear end of T-SCF because of the high loss. The output powers of $\mathrm{LP}_{11}$ from the concave, linear, and convex T-SCF are $1.471 .68 \times 10^{-9}, 0.00016$, and $0.083 \mathrm{~W}$. Amplification characteristics of $\mathrm{LP}_{31 \mathrm{e}}$ are given in Figure 14(b). Signal of 
$\mathrm{LP}_{31 \mathrm{e}}$ is amplified to several tens of watts and then shows a slight decline. The power content of HOMs is relatively high under the large-to-small amplification scheme when considering both $\mathrm{LP}_{11}$ and $\mathrm{LP}_{31 \mathrm{e}}$. Comparing Figure 8 with Figure 14, it can be concluded that the small-to-large scheme is better at filtering out HOMs.

Figure 15 compares the heat load density of the concave TSCF between the two amplification schemes. The maximum heat load density of the concave T-SCF under the large-tosmall scheme is $1.355 \times 10^{10} \mathrm{~W} / \mathrm{m}^{3}$, whereas that under the small-to-large scheme is four times higher. This comparison clearly shows that the large-to-small amplification scheme can mitigate the heat density.

Keeping the bending radius as $32 \mathrm{~cm}$, we investigate the bending-induced HOMs filtering effects under the large-tosmall pumping scheme. As illustrated in Figure 16(a), $\mathrm{LP}_{11}$ still is effectively amplified in the middle section of T-SCF. The maximum power of $\mathrm{LP}_{11}$ in concave, linear, and convex $\mathrm{T}$-SCF is $7.55,9.88$, and $12.36 \mathrm{~W}$, respectively, keeping the power content high. However, the power of $\mathrm{LP}_{11}$ gradually decreases in the rear section of the T-SCF owing to its high loss. The output power of $\mathrm{LP}_{11}$ in concave, linear, and convex $\mathrm{T}$-SCF is $7.69 \times 10^{-10}, 2.54 \times 10^{-10}, 0.00557 \mathrm{~W}$. The $\mathrm{LP}_{11}$ mode can be considered to be effectively stripped off because the output power of $\mathrm{LP}_{11}$ is extremely low, but the effective

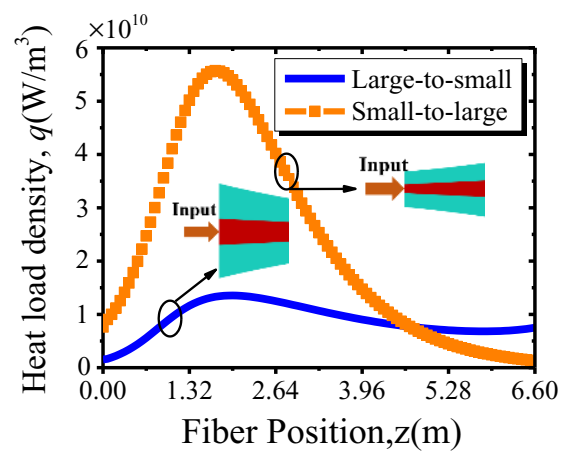

Figure 15. Comparison of heat load density between the two amplification schemes. amplification of $\mathrm{LP}_{11}$ in the middle section of T-SCF may degrade the amplification efficiency of $\mathrm{LP}_{01}$. Figure 16(b) indicates that the $\mathrm{LP}_{31 \mathrm{e}}$ mode can be filtered out under the large-to-small scheme. According to the analyses above, it can be shown that the large-to-small scheme can decrease the heat load density and maintain HOMs filtering effect when compared with the small-to-large scheme.

\section{Conclusion}

In conclusion, we have proposed a potential T-SCF with LMA. Mode and amplification characteristics of the TSCF with different tapered structures have been investigated by FEM and few-mode steady-state rate equation. Simulation results suggest that the concave tapered structure has advantages in stripping off HOMs, whereas the convex tapered structure provides LMA and low heat load density. In addition to tapered structures, the bending operation and amplification scheme have effects on the laser and thermal performance. The $6.6 \mathrm{~m}$ long T-SCF under a bending radius of $32 \mathrm{~cm}$ can achieve single-mode operation when choosing the small end of the fiber as the signal input end, but it keeps relatively high content of $\mathrm{LP}_{11}$ power when choosing the large end of the fiber as the signal input end. Moreover, heat load density under the large-to-small amplification scheme is four times lower than that under the small-tolarge amplification scheme. These results suggest that different tapered structures and amplification schemes present different advantages on achieving single-mode operation and mitigating nonlinear effects as well as thermal effects, which provides theoretical suggestions for designing high-power fiber lasers and amplifiers.

\section{Acknowledgments}

This work was jointly supported by the National Key R\&D Program of China (No. 2020YFB1805802), National Natural Science Foundation of China (Nos. 62005012 and 61827817), State Key Laboratory of Rail Traffic Control and Safety (No.
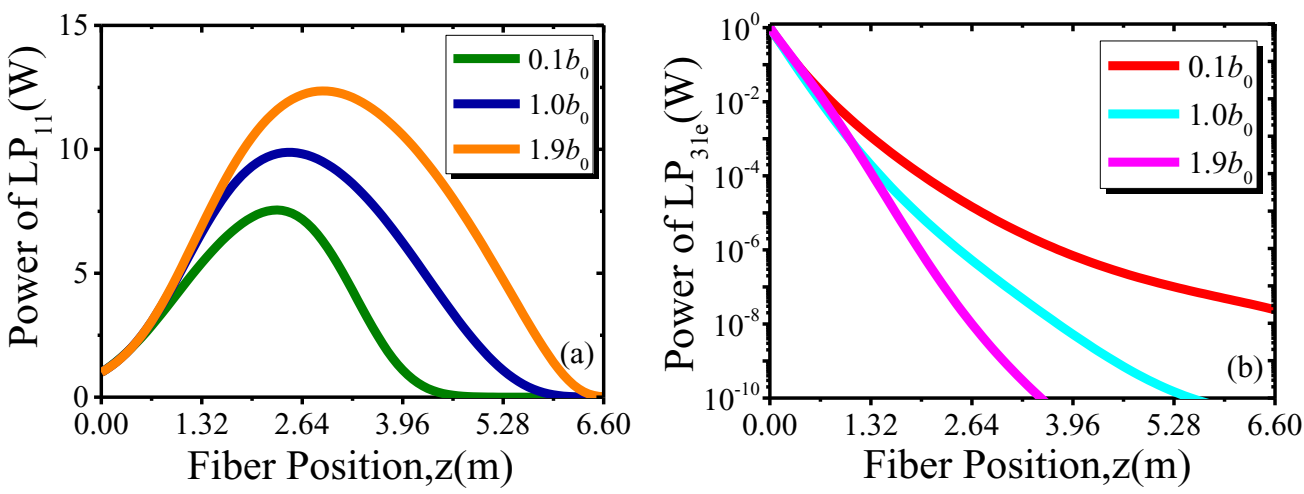

Figure 16. Power of (a) $\mathrm{LP}_{11}$ and (b) $\mathrm{LP}_{31 \mathrm{e}}$ of the $\mathrm{T}-\mathrm{SCF}$ under a bending radius of $32 \mathrm{~cm}$. 
RCS2019ZZ007), Beijing Jiaotong University, and Shandong Province Higher Educational Science and Technology Program (No. J18KA368).

\section{References}

1. A. Tünnermann, T. Schreiber, F. Röser, A. Liem, S. Höfer, H. Zellmer, S. Nolte, and J. Limpert, J. Phys. B 38, S681 (2005).

2. S. Ikoma, H. K. Nguyen, M. Kashiwagi, K. Uchiyama, K. Shima, and D. Tanaka, Proc. SPIE 10083, 100830Y (2017).

3. C. Jauregui, J. Limpert, and A. Tünnermann, Nat. Photonics 7, 861 (2013).

4. J. Limpert, F. Roser, S. Klingebiel, T. Schreiber, C. Wirth, T. Peschel, R. Eberhardt, and A. Tiinnermann, IEEE J. Sel. Top. Quantum Electron. 13, 537 (2007).

5. C. Liu, J. Liu, Y. Zhang, Y. Hou, S. Qi, X. Feng, and P. Wang, Opt. Express 25, 9569 (2017).

6. D. Nodop, C. Jauregui, F. Jansen, J. Limpert, and A. Tünnermann, Opt. Lett. 35, 2982 (2010).

7. D. J. Richardson, H. L. Offerhaus, and N. G. R. Broderick, in Advanced Solid State Lasers (Optical Society of America, 2000), paper MD1.

8. R. A. Morandotti, M. Jäger, H. E. Ruda, S. Caplette, P. Verville, J. Yao, and A. Villeneuve, Proc. SPIE 5971, 59710N (2005).

9. L. Huang, T. Yao, J. Leng, S. Guo, R. Tao, P. Zhou, and X. Cheng, Appl. Opt. 56, 5412 (2017).

10. A. Kumar, T. S. Saini, K. D. Naik, and R. K. Sinha, Appl. Opt. 55, 4995 (2016).

11. G. Gu, F. Kong, T. W. Hawkins, P. Foy, K. Wei, B. Samson, and L. Dong, Opt. Express 21, 24039 (2013).

12. D. Jain, C. Baskiotis, and J. K. Sahu, Opt. Express 21, 1448 (2013).

13. D. Jain, Y. Jung, P. Barua, S. Alam, and J. K. Sahu, Opt. Express 23, 7407 (2015).

14. V. Rastogi and K. S. Chiang, Opt. Lett. 26, 491 (2001).

15. S. Ma, T. Ning, S. Lu, J. Zheng, J. Li, and L. Pei, J. Lightwave Technol. 36, 2844 (2018)

16. D. Jain, C. Baskiotis, T. C. May-Smith, J. Kim, and J. K. Sahu, IEEE J. Sel. Top. Quantum Electron. 20, 242 (2014).
17. J. W. Dawson, M. J. Messerly, J. E. Heebner, P. H. Pax, and M. Dubinskii, Proc. SPIE 7686, 768611 (2010).

18. A. Yeung, K. S. Chiang, V. Rastogi, P. L. Chu, and G. D. Peng, in Optical Fiber Communication Conference (Optical Society of America, 2004), paper ThI4.

19. B. Hooda, A. Pal, V. Rastogi, R. Sen, J. Gandhi, and J. Kobelke, Opt. Eng. 54, 075103 (2015).

20. M. Y. Koptev, E. A. Anashkina, K. K. Bobkov, M. E. Likhachev, A. E. Levchenko, S. S. Aleshkina, S. L. Semjonov, A. N. Denisov, M. M. Bubnov, D. S. Lipatov, A. Y. Laptev, A. N. Gur'yanov, A. V. Andrianov, S. V. Muravyev, and A. V. Kim, Quantum Electron. 45, 443 (2015).

21. J. Ballato, V. Filippov, Y. K. Chamorovskii, K. M. Golant, A. Vorotynskii, and O. G. Okhotnikov, Proc. SPIE 9728, 97280V (2016).

22. K. Bobkov, A. Andrianov, M. Koptev, S. Muravyev, A. Levchenko, V. Velmiskin, S. Aleshkina, S. Semjonov, D. Lipatov, A. Guryanov, A. Kim, and M. Likhachev, Opt. Express 25 , 26958 (2017).

23. A. Fedotov, T. Noronen, R. Gumenyuk, V. Ustimchik, Y. Chamorovskii, K. Golant, M. Odnoblyudov, J. Rissanen, T. Niemi, and V. Filippov, Opt. Express 26, 6581 (2018).

24. B. Yang, H. Zhang, C. Shi, X. Wang, Z. Pan, Z. Wang, P. Zhou, and X. Xu, Opt. Express 27, 7585 (2019).

25. S. Ma, T. Ning, J. Li, J. Zheng, X. Wen, and L. Pei, Opt. Laser Technol. 88, 172 (2017).

26. A. W. Al-Alimi, M. H. Abu Bakar, N. H. Zainol Abidin, A. F. Abas, M. T. Alresheedi, and M. A. Mahdi, Opt. Laser Technol. 112, 26 (2019).

27. O. G. Okhotnikov, Fiber Lasers (Wiley-VCH, 2012).

28. Y. Tian, Y. Chen, J. Leng, T. Yao, P. Zhou, and J. Chen, Opt. Commun. 423, 6 (2018).

29. C. Shi, X. Wang, P. Zhou, X. Xu, and Q. Lu, Opt. Express 24, 19473 (2016).

30. J. Han, G. Gao, Y. Zhao, and S. Hou, J. Lightwave Technol. 35, 2526 (2017).

31. D. Jain, M. Nunez-Velazquez, and J. K. Sahu, Opt. Express 22, 31078 (2014)

32. C. Jauregui, C. Stihler, and J. Limpert, Adv. Opt. Photonics 12, $429(2020)$

33. M.-J. Li, A. Liu, S. Gray, J. Wang, D. T. Walton, and L. A. Zenteno, J. Lightwave Technol. 27, 3010 (2009). 